\title{
Inhalt des zweiten Bandes
}

\author{
Seite \\ III/1 Personenbeförderungsgesetz . . . . . . . . . 1-118 \\ Full \\ (PBefG v. 21. 3. 1961 [BGBl. I, 241] mit Änderungen \\ v. 1965 und 1968) \\ III/1 Grenzüberschreitender Personenverkehr mit \\ Kraftomnibussen. . . . . . . . . 95- 98 Full \\ zu $\$ 52$ (V Nr. 117/66/EWG des Rates v. 28. 7. 1966 über die \\ Eiinführung gemeinsamer Regeln [Amtsbl. Europ. Ge- \\ meinschaften 1966, 2688]) \\ III/1 Freistellungsverordnung ........ 103-104 Full \\ zu $\$ 58$ (Verordnung über die Befreiung bestimmter Beförde- \\ rungsfälle von den Vorschriften des Personenbeförde- \\ rungsgesetzes) $\nabla .30 .8$. 1962 (BGBl. I, 601) mit ände- \\ rung 1967
}

Bearbeiter

III/1 Allgemeine Verwaltungsvorschrift

zum Personenbeförderungsgesetz. . . . . 105-108 Full

zu $§ 59$ v. 2. 7. 1962 (BAnz. Nr. 128 S. 1)

III/2 BOKraft ............. 119-151 Full

(Verordnung über den Betrieb von Kraftfahrunternehmen im Personenverkehr) v. 7. 7. 1960 (BGBl. I, 553) mit Änderungen v. 1963, 1966, 1968 und 1969

III/3

BOStrab . . . . . . . . . . . 152-212

Full

(Verordnung über den Bau und Betrieb der Straßenbahnen; Straßenbahn-Bau- und Betriebsordnung) $\nabla$. 31. 8. 1965 (BGBI. I, 1513) mit Änderung v. 1968

III/3 Anweisung über Haltestellenzeichen . . . . 205-210 Full

zu An- (Anweisung zur Einführung einheitlicher Haltestellen-

lage 4 zeichen für Straßenbahnen und Kraftlinien) v. 19.7. 1959 (DRAnz. Nr. 172)

IV Verordnung über Garagen und Einstellplätze . 213-230 Rüth (GarO) v. 17. 2. 1939 (RGBl. I, 219)

V/1 Kraftfahrzeug-Haftpflichtversicherung, . . . 231-232 Rüth allgemeine Grundsätze

V/2 Gesetz zur Änderung von Vorschriften .... 232-233 Rüth über die Pflichtversicherung für Kraftfahrzeughalter จ. 5. 4. 1965 (BGBl. I, 213) (Auszug) 
Inhalt

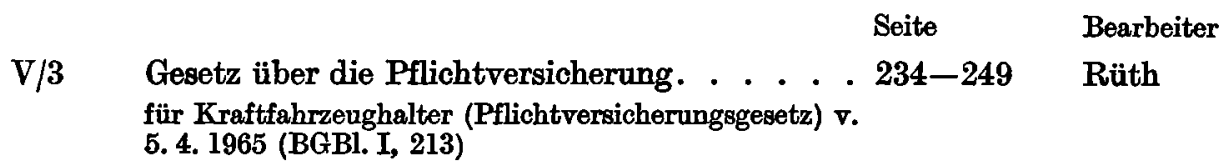

V/4 Gesetz über die Haftpflichtversicherung für aus-

ländische Kraftfahrzeuge . . . . . . . 249-253 Rüth

und Kraftfahrzeuganhänger v. 24. 7. 1956 BGBl.I, 667;

berichtigt BGBl. $1957 \mathrm{I}, 368$ ) mit Änderungen 1964 und

1965

V/5 Schadensfälle mir ausländischen Kraftfahrern . 253-255 Rüth

im In- und Ausland (BMV v. 10. 4. 64, VkBl. 1964, 191)

V/6 Verordnung über den Entschädigungsfonds . . 255-256 Rüth

für Schäden aus Kraftfahrzeugunfällen v. 14. 12. 1965

(BGBl. I, 2093)

V/7 Pflichtversicherung für Angehörige der Alliierten

Streitkräfte . . . . . . . . . . 256 Rüth

V/8 Bekanntmachung über die Änderung von Bedingungen in der Kraftverkehrsversicherung . . .

$257 \quad$ Rüth

(Bekanntmachung des Bundesaufsichtsamts für das Versicherungs- und Bausparwesen) v. 3. 9. 1965 (BAnz. Nr. 172 v. 14.9. 1965)

V/8 Anordnung über die Anderung der Bedingungen in der Kraftverkehrsversicherung . . . . . . v. 15. 9. 1965 (BAnz. Nr. 176 v. 18. 9. 1965)

V/10 Allgemeine Bedingungen für die Kraftverkehrsversicherung. . . . . . . . . . . . 258-289 Rüth (AKB) (BAnz. Nr. 172 v. 14. 9. 1965)

V/11 Gesetz über den Versicherungsvertrag . . . 289-320 Rüth (VVG) v. 30. 5. 1908 (RGBl. 263) i. d. F. v. 5. 4. 1965 (BGBl. I, 213)

VI Sachschadenhaftpflichtgesetz ....... 321-340 Full (Gesetz über die Haftpflicht der Eisenbahnen und Straßenbahnen für Sachschäden) (SHG) v. 29.4. 1940 (RGBl. I, 691) i. d. F. v. 16. 7. 1957 (BGBl. I, 710)

VII/la Internationales Abkommen über den Verkehr mit Kraftfahrzeugen . . . . . . . . 341-351 Rüth vom 24. 4. 1926 (RGBl. $1930 \mathrm{II}, 1233$ ) 
VII/1 b Gesetz zu dem Ubbereinkommen vom 20. März 1857 über die Annahme einheitlicher Bedingungen für die Genehmigung der Ausrüistungsgegenstände und Teile von Kraftfahrzeugen . . . 352-360 Rüth und über die gegenseitige Anerkennung der Genehmigung (vom 12.6. 1965, BGBl. II, $857 \doteq \mathrm{VkBl}$. 387)

VII/1c Utbersicht über die zulässigen Abmessungen, Achslasten und Gesamtgewichte . . . . . 361-367 Rüth der Straßenfahrzeuge vom 4. 6. 1963 ( $\mathrm{VkBl} .1963,259)$

VII/2 Verordnung über internationalen Kraftfahrzeugverkehr ............. 368-409 Rüth (IntVO) v. 12.11.1934 (RGBl. I, 1137) mit Änderungen von $1935,1936,1937$ und 1940

VII/3 Internationales Abkommen über den Straßenverkehr ............... 410 Rüth v. 19. 9.1949 (noch nicht in Kraft)

VII/4 Gesetz zu dem Europäischen Ubbereinkommen $\nabla$. 13. 12. 1957 über Straßenmarkierungen . . . 411-412 Rüth v. 29. 6. 1962 (VkBl. 1962, 373)

VII/5 Zwischenstaatliche Vereinbarungen der Nordatlantikpakt-Mächte über die Rechtsstellung ihrer Truppen . . . . . . . . . . . . 413

Rüth

$\mathrm{VII} / 5 \mathrm{a}$ NTS - AG . . . . . . . . . . . . 414-419 Rüth

(Gesetz zu dem Abkommen zwischen den Parteien des Nordatlantikvertrags vom 19. 6. 1951 über die Rechtsstellung ihrer Truppen und zu den Zusatzvereinbarungen vom 3. 8. $1959 \mathrm{zu}$ diesem Abkommen) v. 18. 8. 1961 (BGBl. II, 1183)

VII/5 b Bekanntmachung über das Inkrafttreten des Abkommens zwischen den Parteien des Nordatlantikvertrags über die Rechtsstellung ihrer Truppen: NATO-Truppenstatut; . . . . . . . 420-421 Rüth und der Zusatzvereinbarungen zu diesem Abkommen v. 16. 6. 1963 (BGBl. 1963 II, 745)

VII/5 c NATO-Truppenstatut . • . • . • • . . . 422-435 Rüth vertrags vom 19.6. 1951 über dio Rechtsstellung ihrer Truppen) (NTS) (BGBl. $1961 \Pi, 1183,1190)$

VII/5d Zusatzabkommen ...........436-463 Rüth zu dem Abkommen zwischen den Parteien des Nordatlantikvertrags über die Rechtsstellung ihrer Truppen hinsichtlich der in der Bundesrepublik Deutschland stationierten ausländischen Truppen (ZA) v. 3. 8. 1959 (BGBl. 1961 II, 1183, 1218) (Auszug) 
Inhalt

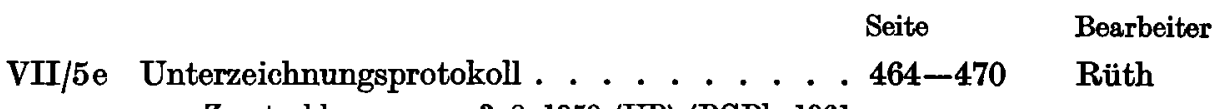
zum Zusatzabkommen v. 3. 8. 1959 (UP) (BGBl. 1961 II, 1313)

VII/6 Verkehrsordnung für die alliierten Streitkräfte 471-472 Rüth (für Berlin West) (VO Nr. 507 der Kommandanten des amerikanischen, britischen und französischen Sektors von Berlin) v. 21. 5. 1951 (GVOBl. 396) mit Änderungen v. 1954, 1956, 1957, 1961 und 1964

VIII/1 Gesetz über die Eingliederung des Saarlandes . 473-478 Rüth v. 23. 12. 1956 (BGBl. I, 1011)

VIII/2 Gesetz zur Einführung von Bundesrecht im Saarland . . . . . . . . . . . . . . . . . 479

Rüth v. 30. 6. 1959 (BGBl. I, 313) (Auszug)

IX/1 Gesetz zur Sicherung des Straßenverkehrs . . . 480-482 Rüth vom 19. 12. 1952 (Erstes VerkSichG) (BGBl. I, 832) mit Änderung v. 1957

IX/2 Zweites Gesetz zur Sicherung des Straßenverkehrs. . . . . . . . . . . . . . . . . . . 483-486 Rüth (Zweites VerkSichG) vom 26. 11. 1964 (BGBl. I, 921)

X Verordnung über Fahrlehrer. . . . . . 487-908 Full im Kraftfahrzeugverkehr (FahrlehrerV) v. 23. 7. 1957 (BGBl. I, 769) mit Änderung v. 1960

XI Selbstfahrerverordnung . . . . . . . 509-512 Rüth (Verordnung über die Überwachung von gewerbsmäßig an Selbstfahrer zu vermietenden Personenwagen und Krafträdern) v. 4. 4. 1955 (BGBl. I, 186)

XII Bundesfernstraßengesetz . . . . . . . 513-530 Full (FStrG) v. 6. 8. 1953 (BGBl. I, 963) mit Änderungen v. 1960, 1961, 1963 und 1968

XIII Kraftfahrsachverständigen-Verordnung . . . 531-540 Rüth (Verordnung über amtlich anerkannte Sachverständige und amtlich anerkannte Prüfer für den Kraftfahrzeugverkehr) v. 10. 11. 1956 (BGBl. I, 855) mit Äderung v. 1960

XIV Gebührenordnung ...........541-549 Rüth für Maßnahmen im Straßenverkehr (GebO) v. 18.5. 1961 (BGBl. I, 611) mit Änderungen von 1966 
Inhalt

$\begin{array}{lll}\text { XV Schichtenbuchverordnung. . . . . . . . 5eite } & \text { Bearbeiter } \\ \text { (Verordnung über Schichtenbücher für Kraftfahrer und } & \text { Rüth } \\ \text { Beifahrer) v. 8. 2.1956 (BGBl. I, 65) } & & \end{array}$

XVI Arbeitszeitordnung ..........555-562 Rüth (AZO) v. 30.4. 1938 (RGBl. I, 447) (Auszug)

zu XVI Ausführungsverordnung zur Arbeitszeitverordnung . . . . . . . . . . . . . . 562-564 Rüth (AVAZO) v. 12. 12. 1938 (BGBl. I, 1799) (Auszug)

XVII Gesetz über die Errichtung eines Kraftfahrt-

Bundesamtes . . . . . . . . . . . 565-566 Rüth v. 4. 8.1951 (BGBl. I, 488) mit Änderung v. 1957

XVIII Eisenbahnkreuzungsgesetz ........567-570 Rüth (Gesetz über Kreuzungen von Eisenbahnen und StraBen) v. 14. 8. 1963 (BGBl. I, 68l)

XIX/1 Gesetz über Ordnungswidrigkeiten . . . . . 571-782 Rüth (OWiG) v. 24. 5. 1968 (BGBl. I, 481)

XIX/2 Einführungsgesetz zum Gesetz über Ordnungswidrigkeiten. . . . . . . . . . 783-796 Rüth (EGOWiG) v. 24. 5. 1968 (BGBI. I, 503)

XIX/3 Bußgeldkataloge der Länder . . . . . . 797-834 Rüth

XIX/4 Verordnungen über die Zuständigkeit . . . 835-842 Rüth für die Verfolgung und Ahndung von Ordnungswidrigkeiten

Register zum zweiten Band . . . . . . . 843-867 
